\title{
Crosstransplantation of Kidneys in Normal and Hyp Mice

\author{
Evidence That the Hyp Mouse Phenotype Is Unrelated to an Intrinsic Renal Defect
}

Teresa Nesbitt, Thomas M. Coffman, Robert Griffiths, and Marc K. Drezner

Departments of Medicine and Cell Biology, and The Sarah W. Stedman Center For Nutritional Studies Duke University Medical Center and Veterans Administration Medical Center, Durham, North Carolina 27710

\begin{abstract}
Although deranged phosphate transport is the fundamental abnormality in X-linked hypophosphatemic (XLH) rickets, it remains unknown if this defect is the consequence of an intrinsic kidney abnormality or aberrant production of a humoral factor. To discriminate between these possibilities, we examined phosphate homeostasis in normal and $H y p$ mice, subjected to renal crosstransplantation. We initially evaluated the effects of uninephrectomy on the indices of phosphate metabolism that identify the mutant biochemical phenotype. No differences were found in the serum phosphorus concentration, fractional excretion of phosphate (FEP), or tubular reabsorption of phosphate per milliliter of glomerular filtrate (TRP) in uninephrectomized normal and Hyp mice, compared with sham-operated controls. Subsequently, single kidneys from normal or $H y p$ mice were transplanted into normal and $H y p$ mouse recipients. Normal mice transplanted with normal kidneys and $H y p$ mice engrafted with mutant kidneys exhibited serum phosphorus, FEP, and TRP no different from those of uninephrectomized normal and Hyp mice, respectively. However, engraftment of normal kidneys in Hyp mice and mutant kidneys in normal mice affected neither serum phosphorus $(4.69 \pm 0.31$ and $8.25 \pm 0.52 \mathrm{mg} / \mathrm{dl}$, respectively) nor FEP and TRP of the recipients. These data indicate that the $H y p$ mouse phenotype is neither corrected nor transferred by renal transplantation. Further, they suggest that the phosphate transport defect in $\mathrm{Hyp}$ mice, and likely $\mathrm{X}$ linked hypophosphatemia, is the result of a humoral factor, and is not an intrinsic renal abnormality. (J. Clin. Invest. 1992. 89:1453-1459.) Key words: hyp mice • rickets • phosphate • kidney
\end{abstract}

\section{Introduction}

$\mathrm{X}$-linked hypophosphatemia $(\mathrm{XLH})^{1}$ is the most common form of inherited vitamin D-resistant rickets/osteomalacia in humans (1). The X-linked hypophosphatemic ( $H y p)$ mouse

Portions of this work have appeared in abstract form in 1990. ( $J$. Bone Miner. Res. 5(2):S205.) Address correspondence and reprint requests to Dr. Teresa Nesbitt, Box 3285, Duke University Medical Center, Durham, NC 27710.

Received for publication 30 July 1991 and in revised form $05 \mathrm{De}$ cember 1991 .

1. Abbreviations used in this paper: FEP, fractional excretion of phosphate; $\mathrm{Pi}$, phosphate; TRP, tubular reabsorption of phosphate per milliliter of glomerular filtrate, XLH, X-linked hypophosphatemia.

J. Clin. Invest.

(C) The American Society for Clinical Investigation, Inc.

0021-9738/92/05/1453/07 \$2.00

Volume 89, May 1992, 1453-1459 serves as a model for the disease, sharing many of the clinical and biochemical abnormalities observed in patients with XLH (2). Although many investigators have identified deranged phosphate $(\mathrm{Pi})$ transport in the renal proximal tubule of the murine homologue as the primary abnormality underlying this disease $(3,4)$, it remains unknown whether this defect is the consequence of an intrinsic kidney abnormality, or results from production of a humoral factor that alters proximal tubule brush border membrane function. Indeed, these possibilities have been inadequately explored, and previous studies have produced conflicting data. Such investigations include those in which in vivo evaluation of phosphate homeostasis, following parabiotic union of normal and $H y p$ mice, favored an hormonal cause (5-7), and those in which comparison of phosphate transport in cell cultures derived from proximal tubules of these animal models indicated the presence of an intrinsic renal abnormality (8-10). Each of these experimental approaches, however, is fraught with significant limitations, and neither permits simultaneous investigation of the independent influences of renal and humoral factors on phosphate metabolism. In contrast, renal crosstransplantation in normal and $H y p$ mice, and subsequent assessment of phosphate transport in normal kidneys, engrafted in a genetically abnormal hormonal/metabolic domain, and kidneys from mutant animals, transplanted into a normal metabolic milieu, permit examination of both possibilities. Therefore, in the present study, we used the technique of crosstransplantation to comprehensively examine if the disordered phosphate transport in XLH results from a primary or secondary disturbance of kidney function.

\section{Methods}

\section{Animals}

Normal C57BL/6J mice were mated with C57BL/6J heterozygous female Hyp mice, as previously described (11). Male Hyp and normal mice obtained from the resultant litters were selected for study at 6-8 wk of age. All animals received an appropriate diet (as described below) and deionized water ad lib. from weaning until the time of study.

\section{Experimental protocols}

CHARACTERIZATION OF THE HYP MOUSE BIOCHEMICAL PHENOTYPE

Initially we developed the biochemical indices that would permit identification of the Hyp mouse phenotype in subsequent studies in which animals were subjected to renal transplantation. From weaning until sacrifice, normal and Hyp mice received a diet containing $0.6 \%$ of both calcium and phosphorus (Teklad Premier Laboratory Diets, Madison, WI). Identical numbers of normal and $H y p$ mice mice were sham operated, leaving both kidneys undisturbed, and at 1-wk intervals thereafter blood obtained via retroorbital sinus puncture for determination of the plasma phosphorus concentration. 4 wk postoperatively, the animals were anesthetized $(65 \mathrm{mg} / \mathrm{kg}$ phenobarbital i.p.), and the fractional excretion of phosphate (FEP) and tubular reabsorption of phosphate per milliliter of glomerular filtrate (TRP) deter- 
mined. At the end of the clearance procedure the animals were euthanized by exsanguination. In addition, phosphate-depleted (normal) mice were evaluated in order to establish the criteria to differentiate hypophosphatemia of differing origins. These normal mice were fed a phosphorus-deficient diet $(0.2 \%$ inorganic phosphorus, $0.6 \%$ calcium; Teklad Premier Laboratory Diets) for 1 wk before phosphate clearance studies.

Evaluation of phosphate clearance. Phosphate clearance was measured by previously established methods (12). After animals were anesthetized with pentobarbital, a silastic catheter $(0.012 \mathrm{in}$. id, $0.025 \mathrm{in}$. od; Dow Corning Corp., Midland, MI) was placed in the left jugular vein for infusion of $\left[{ }^{14} \mathrm{C}\right]$ Inulin (New England Nuclear, Boston, MA). The right carotid artery was catheterized with tubing (PE-10; Clay Adams, Parsippany, NJ) for electronic monitoring of blood pressure (Comfort Electronics, Durham, NC) and arterial blood collection. In addition, a catheter (PE-50; Clay Adams) was inserted through a suprapubic incision into a nick in the bladder wall for urine collection. The catheter was secured with a single ligature, and the abdominal wall and skin closed to prevent dehydration. After volume replacement with normal saline (2\% body wt over $20 \mathrm{~min}$ ), a priming dose of $\left[{ }^{14} \mathrm{C}\right]$ Inulin was given, followed by a continuous infusion to maintain constant blood levels. After a 30 -min equilibrium period, the clearance of $\left[{ }^{14} \mathrm{C}\right]-$ Inulin was measured during two successive 30 -min periods. Arterial blood was obtained at the midpoint of each collection period, and urine at the conclusion of each time period. Subsequently, the glomerular filtration rate (GFR) was calculated from the concentration of $\left[{ }^{14} \mathrm{C}\right]-$ Inulin in the urine and serum samples $\left(\left[{ }^{14} \mathrm{C}\right]\right.$ Inulin $_{\text {urine }} \times$ Volume $_{\text {urined }} /$ $\left[{ }^{14} \mathrm{C}\right]$ Inulin $_{\text {serum }}$ per min per $\mathrm{kg}$ ). In addition, the serum and urine concentrations of phosphorus were measured in preinfusion samples in order to calculate FEP and TRP according to the following formulas:

FEP $=U_{\mathrm{Pi}} X U_{\text {Volume }} /$ Filtered Volume $\times \mathrm{S}_{\mathrm{Pi}}$ where

Filtered Volume $=$ GFR (milliliter/minute per kidney per kilogram)

$\times \mathrm{BW}(\mathrm{kg}) \times$ minutes $\times($ no. kidneys $)$

$\mathrm{TRP} / \mathrm{ml}$ GFR $=$ Filtered Load $\mathrm{P}_{\mathrm{i}}-\mathrm{U}_{\mathrm{Pi}} / \mathrm{GFR}$ where

Filtered Load $=\mathrm{S}_{\mathrm{Pi}} \times$ GFR (milliliter/minute per kidney per

kilogram) $\times$ BW (kilogram) $\times$ minutes $\times($ no. kidneys $)$

EVALUATION OF RENAL TRANSPLANTATION AND CROSSTRANSPLANTATION

Normal and $H y p$ mice were engrafted with a single kidney from either animal model. Appropriate controls for these studies were obtained by subjecting mice of both genotypes to a sham surgical procedure, leaving both kidneys undisturbed, and a right uninephrectomy, to mimic the single kidney model used in the transplantation studies. Throughout the study, all animals received a diet containing $0.6 \%$ calcium and phosphorus. Blood was obtained from the retroorbital sinus at 1-2 wk intervals after surgery for the determination of serum phosphorus levels. In addition, 4 wk postoperatively, the mice were anesthetized (65 $\mathrm{mg} / \mathrm{kg}$ pentobarbital, i.p.), and the FEP and TRP, as well as the GFR, were determined, as described above.

Renal transplantation technique. In renal transplantation studies we engrafted a single kidney into recipients that were subsequently nephrectomized. We used an end to side, aorta to aorta anastomosis for the renal transplantation, using techniques previously established in rats (13). Donor mice were anesthesized with Nembutal $(50 \mathrm{mg} / \mathrm{kg}$ i.p.), and the aorta and vena cava separated and dissected from a point distal to the left renal vessels to an area proximal to the right renal vessels. Three ligatures were preplaced around $(a)$ the aorta and vena cava, distal to the left kidney, and $(b)$ the vena cava and aorta individually, distal to the right renal vessels. The left ureter was dissected to its insertion in the bladder wall, the right ureter was cauterized and severed, and a ligature was preplaced around the urethra. The donor mouse was then set aside.

Recipient mice were anesthetized with $4 \%$ Halothane and maintained with a concentration of $1.5 \%$. Heparin $(1,000 \mathrm{U} / \mathrm{ml}$; Elkins-Sinn
Inc., Cherry Hill, NJ) was administered to both recipient and donor mice. After a left nephrectomy, the abdominal aorta and vena cava were dissected and separated, and the recipient mouse was set aside.

The donor kidney was obtained by ligating the preplaced ligatures and severing the vessels/urethra distal to the ties. The kidney, urethra, and bladder were then placed in an ice-cold solution of $0.1 \mathrm{ml}$ heparin/ $20 \mathrm{ml}$ stock solution $(4.5 \mathrm{~g}$ sodium chloride, $100 \mathrm{~g}$ mannitol, and $1 \mathrm{~g}$ Chloromycetin Sodium/liter sterile water).

In the recipients, vascular clamps were placed proximally and distally in preparation for aorotomy and venotomy. The free proximal ends of the donor aorta and vena cava were sutured to the respective recipient aorta and vena cava with 11-0 Dermalon, and the vascular clamps removed. The proximal $1 / 5$ of the recipient bladder and the proximal $2 / 3$ of the donor bladder were excised in preparation for creating a new urinary bladder in the transplanted mouse. The remaining $1 / 3$ of the donor bladder with its attatched ureter was sutured to the remaining $4 / 5$ of the recipient bladder, using 10-0 Novafil.

The abdominal wall and skin were closed separately with continuous sutures of 4-0 silk. $4 \mathrm{~d}$ after transplantation, the engrafted mouse was anesthetized with Halothane and a nephrectomy performed on the remaining native kidney.

\section{Analytical methodology}

Urine volume was determined by weighing. Plasma (14) and urine (15) inorganic phosphorus were determined by a colorimetric technique.

\section{Statistical methods}

All results are expressed as the mean \pm SE. Significance of the difference between groups was evaluated using paired $t$ testing and analysis of variance (with appropriate a posteriori multiple range comparison procedures) (16). 4-10 animals comprised each experimental group.

\section{Results}

Characterization of the Hyp mouse biochemical phenotype The serum phosphorus concentration in $H y p$ and phosphatedepleted mice, although characteristically decreased to a level significantly less than that in normals, did not distinguish these animal models (Fig. 1). However, Hyp mice exhibited an FEP far in excess of that manifested by phosphate-depleted mice, and a TRP that was twofold less than that in either normals or phosphate-depleted normals (Fig. 1). Collectively, these observations established that the decreased circulating level of phosphorus in $H y p$ mice is uniquely due to renal phosphate wasting. Therefore, identification of the $H y p$ mouse phenotype in transplanted or uninephrectomized animals not only requires documentation of persistent hypophosphatemia, but the presence of a normal FEP and a decreased TRP, evidence of abnormal renal phosphate handling.

\section{Effects of uninephrectomy, renal transplantation, and renal crosstransplantation on phosphate homeostasis}

Uninephrectomy. Initially, we performed uninephrectomy in normal and $H y p$ mice to determine the effects of a single kidney on the indices of phosphate homeostasis that identified the mutant biochemical phenotype. $4 \mathrm{wk}$ postoperatively, body weight and GFR (per kidney) in both uninephrectomized normal and Hyp mice were no different than those of their respective sham operated controls (Fig. $2 \mathrm{~A}$ ). Similarly, uninephrectomy had no effect on the plasma phosphorus values in either animal model (Fig. $3 \mathrm{~A}$ ), and the presence of a single kidney did not significantly alter the measures of renal phosphate handling, FEP and TRP (Fig. $4 A$ ). These data indicate that the experimental model of a single transplanted kidney 

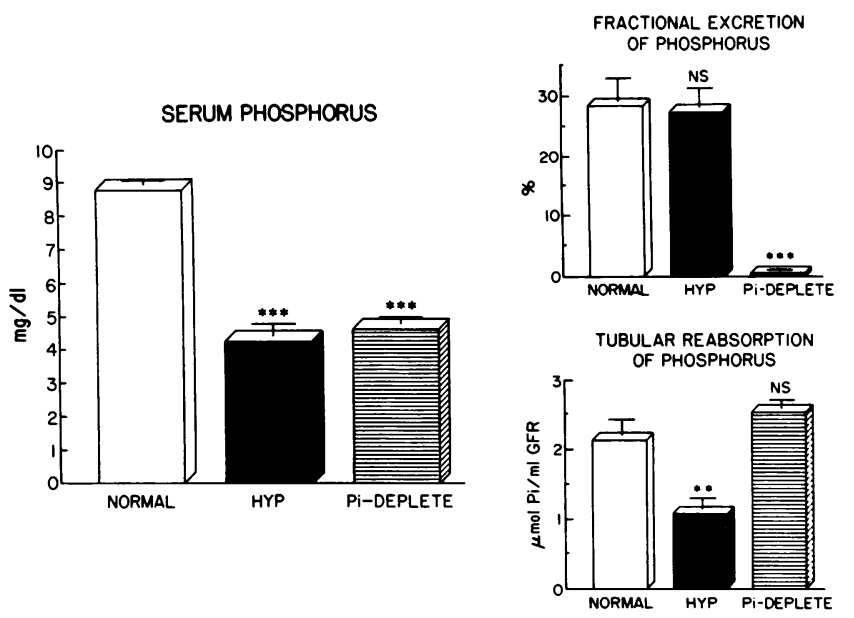

Figure 1. Serum phosphorus concentration, fractional excretion of phosphorus, and tubular reabsorption of phosphorus in sham-operated normal, $H y p$, and phosphate-depleted mice. Serum phosphorus levels in Hyp mice were not different from those in Pi-depleted mice, although they were significantly lower than those in normal mice $(P$ $\leq 0.001)$. The fractional excretion of phosphorus was no different in normal and $H y p$ mice but markedly reduced in Pi-depleted mice ( $P$ $\leq 0.001$ ). The tubular reabsorption of phosphorus was significantly reduced in $H y p$ mice $(P \leq 0.01)$, compared with normal mice, while Pi-depleted animal models displayed TRP levels no different from those in normals. Data are expressed as mean \pm SEM of 6-10 individual determinations per group.

which we used in subsequent studies had no effect on $\mathrm{P}_{\mathrm{i}}$ homeostasis.

Renal transplantation. Likewise, renal transplantation between a donor and recipient of the same genotype did not influence body weight or GFR (Fig. $2 \mathrm{~B}$ ). Moreover, this procedure did not affect phosphate homeostasis. 4 wk after surgery, normal mice engrafted with a normal kidney maintained a plasma phosphorus level significantly greater than $H y p$ mice transplanted with a mutant kidney (Fig. $3 \mathrm{~B}$ ), a relationship identical to that observed in both sham-operated and uninephrectomized animals. Indeed, throughout the postoperative interval, the engrafted normal mice did not vary their plasma phosphorus $(9.6-10.8 \mathrm{mg} / \mathrm{dl})$ from the normal range, while transplanted $H y p$ mice exhibited a persistently decreased plasma phosphorus, 5.4-6.4 mg/dl, (Fig. $5 A$ ). Renal transplantation, likewise, did not alter the dynamics of renal phosphate handling in normal or $H y p$ mice. Normal mice engrafted with normal kidneys and $H y p$ mice transplanted with mutant kidneys maintained FEP and TRP values comparable to those in their respective sham-operated controls (Fig. $4 \mathrm{~B}$ ). Thus, the FEP in the transplanted mutants was no different from that of engrafted normals, in spite of a remarkably diminished filtered phosphate load, and the TRP was twofold reduced (Fig. $4 \mathrm{~B}$ ). These observations indicate that a single normal and $H y p$ mouse kidney transplant functions in normal and mutant recipients, respectively, to maintain phosphorus homeostasis and the distinctive biochemical profiles of the host animal models.

Renal crosstransplantation. In subsequent studies we investigated the effects of renal crosstransplantation in normal and $H y p$ mice with donors and recipients of different genotypes. 4 wk after surgery, normal mice transplanted with $H y p$ mouse kidneys and Hyp mice engrafted with normal kidneys exhibited body weights and GFRs no different than their sham-oper-

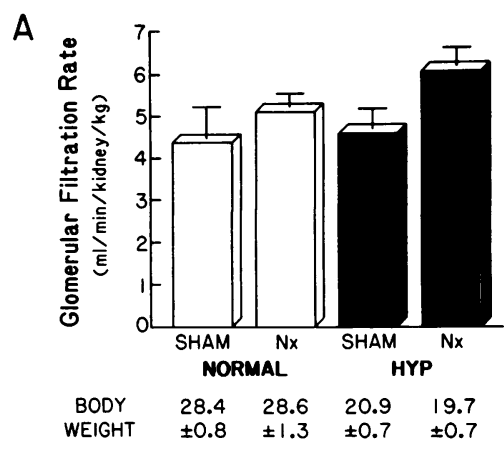

Figure 2. (A) GFR and body weights in sham and uninephrectomized normal and $H y p$ mice $4 \mathrm{wk}$ after the surgical procedure. The GFR was no different in each of these animal models. In contrast, body weights of normal mice were significantly greater than Hyp mice, whether sham operated

B

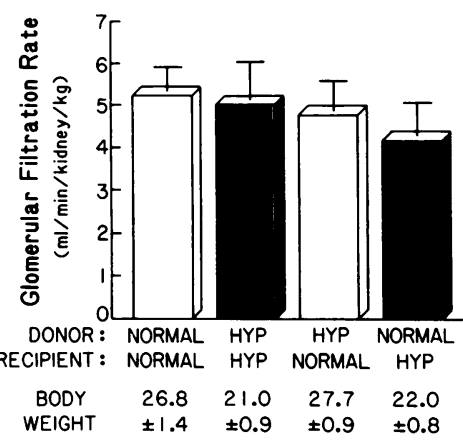
or uninephrectomized (Nx) $(P \leq 0.001)$. However, uninephrectomy had no effect on this variable in normal or $H y p$ mice. Data are expressed as mean \pm SEM of four to six individual determinations per group. (B) GFR and body weights in normal and $H y p$ mice 4 wk after renal transplantation or renal crosstransplan-

tation. No significant differences in GFR were present in any of the groups of mice. Body weights of normal mice were significantly greater than those of $H y p$ mice $(P \leq 0.01)$, regardless of the transplanted kidney type. Data are expressed as mean \pm SEM of four to six individual determinations per group.

A

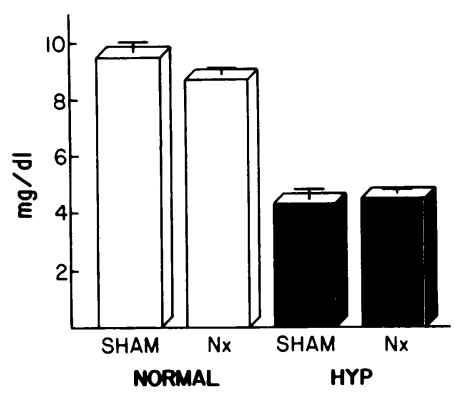

B

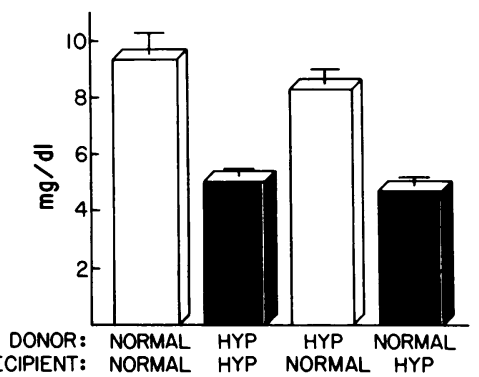

Figure 3. (A) Serum phosphorus concentrations in sham and uninephrectomized (Nx) normal and Hyp mice 4 wk after the surgical procedure. Uninephrectomy had no significant effect on serum phosphorus levels in either normal or Hyp mice. However, both shamoperated and uninephrectomized Hyp mice were significantly hypophosphatemic relative to their respective normals $(P<0.001)$. Data are expressed as mean \pm SEM of 6-10 individual determinations per group. (B) Serum phosphorus concentrations in normal and hyp mice 4 wk after renal transplantation or renal crosstransplantation. Serum phosphorus levels were reflective of whether the recipient was a normal or $H y p$ mouse, and were unrelated to the donor mouse model. Thus, Hyp mouse recipients (black bars) displayed a significant hypophosphatemia $(P<0.001)$, compared with normal mouse recipients (white bars), regardless of whether the transplanted kidney was donated by a normal or Hyp mouse. Data are expressed as mean \pm SEM of four to six individual determinations per group. 

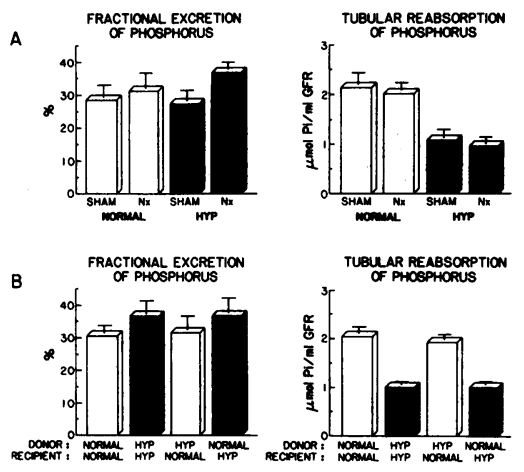

Figure 4. (A) Fractional excretion of phosphorus and tubular reabsorption of phosphorus in sham and uninephrectomized normal and Hyp mice 4 wk after the surgical procedure. Uninephrectomy had no effect on either parameter in both normal and $H y p$ mouse models. In contrast, while shamoperated and unine-

phrectomized normal and Hyp mice displayed no difference in the fractional excretion of phosphorus, these $H y p$ mouse models had a significantly reduced $(P<0.001)$ tubular reabsorption of phosphorus compared with normals. Data are expressed as mean \pm SEM of 6-10 individual determinations per group. $(B)$ Fractional excretion of phosphorus and tubular reabsorption of phosphorus in normal and Hyp mice 4 wk after renal transplantation or renal crosstransplantation. The fractional excretion of phosphorus was not significantly different in any group. However, the tubular reabsorption of phosphorus was reflective of whether the recipient was a normal or $H y p$ mouse, and was unrelated to the donor mouse model. Thus, $H y p$ mouse recipients (black bars) displayed a TRP that was significantly less than normal mouse recipients (white bars), regardless of whether the transplanted kidney was donated by a normal or Hyp mouse ( $P$ $<0.001)$. Data are expressed as mean \pm SEM of four to six individual determinations per group.

A

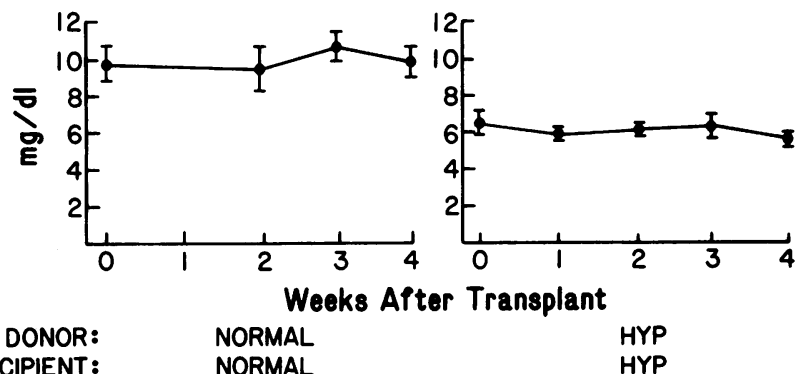

RECIPIENT:

NORMAL

HYP

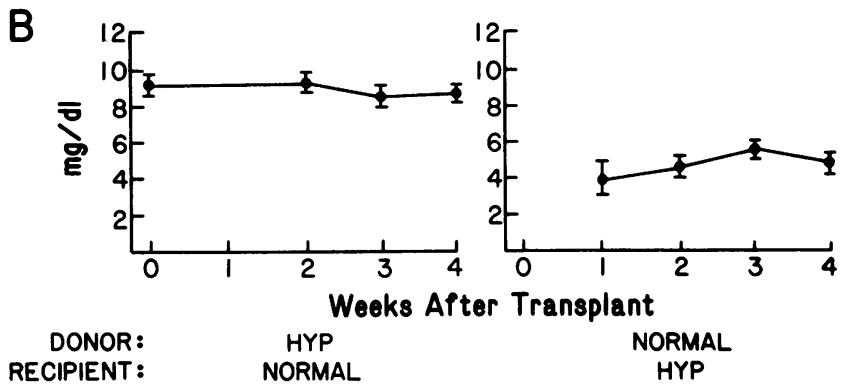

Figure 5. (A) Weekly serum phosphorus levels in normal and $H y p$ mice after renal transplantation. Serum phosphorus levels did not change significantly in the 4-wk postsurgical interval in either normal or Hyp mouse recipients. Data are expressed as mean \pm SEM of four to five individual determinations per group. $(B)$ Weekly serum phosphorus levels in normal and $H y p$ mice after renal crosstransplantation. Normal mice engrafted with $H y p$ mouse kidneys maintained normal serum phosphorus levels throughout the postoperative period. Likewise, Hyp mice crosstransplanted with normal kidneys maintained hypophosphatemia characteristic of the mutant phenotype 4 wk after the surgical procedure. Data are expressed as mean \pm SEM of five to six individual determinations per group. ated or uninephrectomized counterparts (Fig. 2 B). Moreover, the normal mice with kidneys from Hyp mice maintained a plasma phosphorus concentration within the normal range and did not develop the characteristic hypophosphatemia of $H y p$ mice (Fig. $3 \mathrm{~B}$ ). In contrast, Hyp mice engrafted with normal kidneys displayed a decreased plasma phosphorus concentration. Furthermore, the inability of crosstransplantation to alter the plasma phosphorus levels of graft recipients was persistent and unwavering. Within $1 \mathrm{wk}$ of surgery, Hyp mice crosstransplanted with normal kidneys continued to exhibit hypophosphatemia which remained steady state throughout the $4 \mathrm{wk}$ of observation (Fig. $5 \mathrm{~B}$ ). Likewise, normal mice engrafted with mutant kidneys maintained normal plasma phosphorus throughout the post-crosstransplantation period.

The normophosphatemia evident in normal mice crosstransplanted with kidneys from $H y p$ mice was associated with evidence of normal renal phosphate handling. Both FEP and TRP in these animals were no different than those of normal mice bearing normal transplants (Fig. $4 \mathrm{~B}$ ). Indeed, these values indicated that the kidney from Hyp mice, resident in the normal host, was fully capable of normal phosphate transport. In contrast, the normal kidney functioning in the mutant milieu exhibited evidence of markedly abnormal renal phosphate transport. Consequently, Hyp mice, crosstransplanted with normal kidneys, displayed an FEP and TRP no different than those of $H y p$ mice engrafted with kidneys from mutant animals (Fig. $4 \mathrm{~B}$ ). Moreover, the TRP of these animals was significantly less than those of the normal mice crosstransplanted with kidneys from $H y p$ mice.

\section{Discussion}

Renal phosphate reabsorption occurs in the proximal convoluted tubule via a sodium-dependent, phosphate cotransporter that mediates apical transport of phosphate from the tubular lumen into the cell (17). The activity of this system is determined by the integrated effects of multiple intrarenal events, and the modifying influence of a variety of hormones, and metabolic and dietary factors. Therefore, decreased phosphate transport in XLH may be due to a genetic defect that results in a primary or extrarenal abnormality(s) which alters the activity of this apical transport system. Such defects may respectively include: $(a)$ a primary modification of the plasma membrane protein(s) that constitutes the $\mathrm{Na}^{+} / \mathrm{Pi}$ cotransporter and/or the intracellular enzymes/cofactors or renal paracellular hormones that regulate its activity; and $(b)$ a hormonal/metabolic imbalance that secondarily disturbs a component of the transport mechanism and results in aberrant function. Until recently, the majority of available evidence suggested that a primary renal abnormality, such as the absence or diminished activity of a transport-specific protein, underlies this disease. Several observations support this possibility: (a) the expressed abnormality in phosphate transport is confined to the kidney $(18-20)$ and is restricted to the proximal convoluted tubule $(21,22)$; and $(b)$ known hormones that regulate this process, such as parathyroid hormone, circulate within the normal range (23-25). These data, however, provide only inferential evidence in favor of a primary renal defect, and considerable controversy persists regarding the target organ for the genetic defect in XLH. Thus, in the present studies we used renal crosstransplantation of kidneys between normal and Hyp mice to directly examine the independent influences of renal and humoral factors on phos- 
phate metabolism in this genetically determined hypophosphatemic state. This experimental approach provided the opportunity to examine the function of kidneys from Hyp mice in a normal hormonal/metabolic milieu and the function of normal kidneys in the abnormal domain of the mutant.

The technique of organ crosstransplantation has been used previously to distinguish whether the phenotypic and/or biochemical characteristics of a variety of genetic diseases result from innate defects in end-organ function and/or systemic hormonal/metabolic influences. Such investigations have identified an intrarenal abnormality as a primary defect in a genetic form of hypertension in the rat (26), and an intrahepatic defect as causal of deranged uric acid metabolism in Dalmation dogs (27). In contrast, alterations in the affected host environment have been determined as the primary cause of resistance to arteriosclerosis in pigs with von Willebrand's disease (28), and as primary or coexistent abnormalities in avian muscular dystrophy (29). Central to each of these investigations is elucidation of definitive criteria to identify the abnormal phenotype under study, and documentation that surgical intervention does not alter their expression. In our studies, we observed that measurement of plasma phosphorus and TRP reproducibly discriminates the Hyp mouse from both normal and phosphate-depleted normal mice (Fig. 1). Moreover, the single kidney model that we employed did not influence expression of these abnormalities in spite of contrary observations in other animal species (30). Indeed, we found that uninephrectomy and/or renal transplantation between matched murine models did not negatively influence the GFR or alter phosphate clearance in the resultant single kidney. Consequently, the serum phosphorus concentration was unchanged in each treated animal model (Fig. 3). The surgical intervention that we used, therefore, did not confound interpretation of data from the crosstransplantation studies.

Thus, in contrast to prevailing opinion, our observations after renal crosstransplantation indicate that the expressed abnormality of phosphate reabsorption in XLH does not result from an intrinsic renal defect, but more likely from an hormonal/metabolic abnormality. In this regard, normal mice transplanted with kidneys from $H y p$ mice maintained a normal plasma phosphorus concentration throughout the postoperative period, and surprisingly, did not develop hypophosphatemia, in spite of functioning grafts from the mutants (Fig. 3). Furthermore, the normophosphatemia was associated with evidence of normal renal phosphate reabsorption, which included FEP and TRP values well within the normal range. In concert, crosstransplantation of a normal kidney into a $H y p$ mouse failed to alter the characteristic hypophosphatemia of the graft recipient. Moreover, the Hyp mice engrafted with normal kidneys displayed FEP and TRP values characteristic of the mutant biochemical phenotype (Fig. 4). All of these changes occurred without variation in the body weight of the host or diminished GFR in the single kidney (Fig. 2). Therefore, these data illustrate that the Hyp mouse phenotype is neither transferred nor corrected by renal transplantation. Rather, the potential influence of crosstransplanted kidneys from normal or Hyp mice is negated, and their function determined by the hormonal/metabolic milieu of the host animal.

Whether aberrant regulation of renal 25-hydroxyvitamin D- $1 \alpha$-hydroxylase activity, an additional kidney abnormality in XLH, similarly occurs as a consequence of an abnormal hormonal/metabolic milieu remains unknown. Nevertheless, since studies in the Hyp mouse have documented that the deranged renal enzyme activity is likely localized to the proximal convoluted tubule, the site of defective phosphate transport, the causes of these abnormalities are undoubtedly linked. Indeed, currently available evidence (31-33) suggests that the enzyme dysfunction is an acquired defect secondary to this abnormality. Thus, the apparent hormonal/metabolic defect in Hyp mice most likely indirectly influences the expressed defect in calcitriol production.

Our data agree with previous observations $(5,6)$, in which the technique of parabiotic union was used to demonstrate that normal mice paired with $H y p$ mice, before and after parathyroidectomy developed hypophosphatemia secondary to a reversible increase in renal phosphate excretion. However, several salient points limit interpretation of these parabiotic studies. In this regard, the serum phosphorus level and the urinary phosphate excretion in the normal parabiont were intermediate between those evident in the complementary Hyp mouse and sham operated normals. Further, normal mice parabiosed to normal mice also exhibited a decrease in serum phosphorus and an increase in urinary phosphate excretion, compared with sham operated normals. Although some of these observations may be the result of an inability to control the crosscirculation rate in a parabiotic union, such confounding alterations in phosphate homeostasis in parabionts may be due to muscle wasting and/or transfer of phosphorus to the phosphate-depleted bones of the mutant. In any case, no data can be generated from study of parabionts regarding the exclusive ability of the kidney from a $H y p$ mouse to maintain or contribute to the abnormal biochemical profile. Thus, the possibility of a combined defect in the end organ and the host environment cannot be ruled out. Indeed, previous experiments indicate that such a dual abnormality may produce the characteristic phenotype in avian myotonic dystrophy (29). These limitations have precluded definitive conclusions about the hormone dependence of XLH.

In any case, our observations provide no information regarding the identity of the abnormal hormonal/metabolic factor that underlies XLH. Although elevated serum parathyroid hormone levels have been reported in Hyp mice (34), this alteration of the metabolic milieu unlikely underlies the abnormal $\mathrm{Pi}$ homeostasis in the mutants. In this regard, parathyroidectomy of Hyp mice does not alter the serum phosphorus concentration (35). By contrast, the recent discovery and biochemical characterization of the paraneoplastic syndrome, tumor-induced osteomalacia, provides substantial evidence that favors the existence of a unique humoral agent. In this acquired disease, hypophosphatemic rickets/osteomalacia occurs secondary to diminished renal tubular reabsorption of phosphate and abnormally regulated calcitriol production (36). Moreover, removal of small tumors leads to prompt restoration of the biochemical abnormalities and healing of the bone disease (37, 38). Consequently, overproduction of a hormone(s), native or ectopic to the tissue of origin, almost certainly underlies this syndrome. The genetic error in XLH, therefore, may result in altered production (or turnover) of such a naturally occurring humoral factor(s).

Moreover, other evidence does exist that supports the possibility of a humoral phosphaturic factor in X-linked hypophosphatemia. Morgan et al. (39) reported that transplantation of a normal kidney into a patient with XLH resulted in continued hypophosphatemia and decreased renal reabsorption of phos- 
phorus. This evidence is tempered by the tendency of transplanted kidneys in humans to function abnormally and exhibit low renal retention of phosphate (40). Further, at the time of study in the posttransplantation period, an abnormal hormonal milieu secondary to renal failure likely persisted, and may have influenced phosphate homeostasis.

Regardless, there are additional data that are difficult to reconcile with a humoral origin for XLH. Renal cells from $H y p$ mice display diminished phosphate uptake (8-10) and abnormal vitamin $D$ metabolism $(8,41)$ after several days in culture, albeit the abnormalities are not as profound as those observed in vivo. Additionally, osteoblasts from Hyp mice, when transplanted to normal mice, produce abnormally mineralizing bone, consistent with expression of the gene effect in this cell type (42). Although several investigators have suggested that the presence of cellular heterogeneity, potential dedifferentiation, or memory retention of in vivo hormonal influences may explain some of these discrepant in vitro observations, insufficient information is available to ascertain the influence of any of these factors. Alternatively, these disparate findings may be the consequence of a unique hormonal effect that results in a blockade of, or failure to express, an essential gene function in a variety of cell types. The absence of the gene effect may induce the well known end-organ effects of $\mathrm{XLH}$, which include abnormal renal phosphate transport and impaired bone mineralization. While culture of such impaired cells, or their transplantation to a foreign environment, would remove the alleged hormonal influence, these techniques may not result in exposure of the cells to essential factors necessary to reinitiate expression of the gene. As a consequence, the abnormalities present in these cells in vivo may be perpetuated in culture, or after transplantation. Further studies are necessary, however, to determine the reason for these apparently contradictory observations.

Nevertheless, the present findings provide the first unequivocal evidence that the kidney is not the target organ for the genetic abnormality that underlies XLH. Indeed, our observations indicate that the Hyp mouse phenotype is neither transferred nor corrected by renal transplantation. Therefore, we postulate that the XLH syndrome likely results from hormonally induced alterations in the function of renal proximal tubule brush border membranes.

\section{Acknowledgments}

The authors thank Chris Best for his expert technical assistance.

This work was supported by National Institutes of Health grants AR-27032, DK-38015, and DK-38108. Dr. Coffman is a Research Associate at the Veterans Administration Hospital.

\section{References}

1. Rasmussen, H., and C. Anast. 1983. Familial hypophosphatemic rickets and vitamin D-dependent rickets. In The Metabolic Basis Of Inherited Disease J. B. Stanbury, J. B. Wyngarden, D. S. Fredrickson, J. L. Goldstein, and M. S. Brown, editors. McGraw Hill, New York. 1743-1773.

2. Eicher, E. M., J. L. Southard, C. R. Scriver, and F. H. Glorieux. 1976 Hypophosphatemia: mouse model for human familial hypophosphatemic (vitamin D-resistant) rickets. Proc. Natl. Acad. Sci. USA. 73:4667-4671.

3. Giasson, S. D., M. G. Brunette, G. Danan, N. Vigneault, and S. Carriere 1977. Micropuncture study of renal phosphorus transport in hyophosphatemic vitamin D resistant rickets mice. Pfluegers Arch. Eur. J. Physiol. 371:33-38.

4. Tenenhouse, H. S., C. R. Scriver, R. R. McInnes, and F. H. Glorieux. 1978. Renal handling of phosphate in vivo and in vitro by the $\mathrm{X}$-linked hypophosphate- mic male mouse: Evidence for a defect in the brush border membrane. Kidney Int. 14:236-244.

5. Meyer, R. A., Jr., M. H. Meyer, and R. W. Gray. 1989. Parabiosis suggests a humoral factor is involved in X-linked hypophosphatemia in mice. J. Bone Miner. Res. 4:493-500.

6. Meyer, R. A. Jr., H. S. Tenenhouse, M. Meyer, and A. H. Klugerman. 1989. The renal phosphate transport defect in normal mice parabiosed to Xlinked hypophosphatemic mice persists after parathyroidectomy. J. Bone Miner. Res. 4:523-532.

7. Marie, P. J., R. Travers, and F. H. Glorieux. 1981. Mineral and skeletal changes in parabiotic normal and hypophosphatemic mice. Clin. Res. 29:414a. (Abstr.).

8. Bell, C. L., H. S. Tenenhouse, and C. R. Scriver. 1988. Primary cultures of renal epithelial cells from X-linked hypophosphatemic (Hyp) mice express defects in phosphate transport and vitamin D metabolism. Am. J. Hum. Genet. 43:293-303.

9. Dobre, C. V, U. M. Alvarez, and K. A. Hruska. 1990. Primary culture of hypophosphatemic proximal tubule cells express defective adaptation to phosphate. J. Bone Miner. Res. 5:S205 (Abstr.).

10. Kinoshita, Y., M. Fukase, M. Nakada, and T. Fujita. 1987. Defective adaptation to a low phosphate environment by cultured renal tubular cells from X-linked hypophosphatemic (Hyp) mice. Biochem. Biophys. Res. Commun. 144:763-769.

11. Lobaugh, B., and M. K. Drezner. 1983. Abnormal regulation of renal 25-hydroxyvitamin D-1 $\alpha$-hydroxylase activity in the X-linked hypophosphatemic mouse. J. Clin. Invest. 71:400-403.

12. Spurney, R. F., P. Ruiz, D. S. Pisetsky, and T. M. Coffman. 1991. Enhanced leukotrine production in murine lupus. Kidney Int. 39:95-105.

13. Coffman, T. M., W. E. Yarger, and P. E. Klotman. 1985. Functional role of thromboxane production by acutely rejecting renal allografts in rats. J. Clin. Invest. 75:1242-1248.

14. Dryer, R. L., A. R. Tammes, and J. I. Routh. 1957. The determination of phosphorus and phosphatase with N-phenylenediamine. J. Biol. Chem. 225:177183.

15. Friedel, R. O., and S. M. Schanberg. 1971. Incorporation in vivo of intracisternally injected ${ }^{33} \mathrm{Pi}$ into phosphorus of rat brain. J. Neurochem. 18:21912200.

16. Neter, J., and W. Wasserman. 1974. Applied Linear Statistical Models. Irwin Inc., Hanswerd, IL. 352 pp.

17. Kinne, R., W. Berner, N. Hoffmann, and H. Murer. 1976. Phosphate transport by isolated renal and intestinal plasma membranes. Adv. Exp. Med. Biol. 81:265-277.

18. Delzer, P. R., and R. A. Meyer, Jr. 1983. Normal milk composition in lactating X-linked hypophosphatemic mice despite continued hypophosphatemia. Calcif. Tissue Int. 35:750-754.

19. Delzer, P. R., and R. A. Meyer, Jr. 1984. Normal handling of phosphate in the salivary glands of X-linked hypophosphatemic mice. Arch. Oral Biol. 29:1009-1013.

20. Tenenhouse, H. S., and C. R. Scriver. 1981. Effect of 1,25-dihydroxyvitamin $D_{3}$ on phosphate homeostasis in the X-linked hypophosphatemic (Hyp) mouse. Endocrinology. 109:658-660.

21. Giasson, S. D., M. G. Brunette, G. Danan, N. Vigneult, and S. Carriere. 1977. Micropuncture study of renal phosphorus transport in hypophosphatemic vitamin D resistant rickets in mice. Pfluegers Arch. Eur. J. Physiol. 371:33-38.

22. Cowgill, L., S. Goldfarb, K. Lau, E. Slatopolsky, and Z. S. Agus.1979. Evidence for an intrinsic renal tubular defect in mice with genetic hypophosphatemic rickets. J. Clin. Invest. 63:1203-1210.

23. Arnaud, C., F. H. Glorieux, and C. R. Scriver. 1971. Serum parathyroid hormone in X-linked hypophosphatemia. Science (Wash. DC). 173:845-847.

24. Fanconi, A., J. A. Fischer, and A. Prader. 1974. Serum parathyroid concentrations in hypophosphatemic vitamin D-resistant rickets. Helv. Paediatr. Acta. 29:187-194.

25. Krohn, H. P., G. Offermann, M. Brandis, J. Broedehl, K. Hanke, and G. Offner. 1977. Occurrence of hyperparathyroidism in children with X-linked hypophosphatemia under treatment with vitamin D and phosphate. Adv. Exp. Med. Biol. 81:345-351.

26. Bianchi, G., E. Niutta, P. Ferrari, P. Salvati, S. Salaardi, D. Cusi, R. Colombo, B. Cesana, G. Tripodi, P. Pati, et al. 1989. A possible primary role for the kidney in essential hypertension. Am. J. Hypertens. 2:2S-6S.

27. Kuster, G., R. G. Shorter, B. Dawson, and G. A. Hallenbeck. 1972. Uric acid metabolism in Dalmations and other dogs. Arch. Intern. Med. 129:492-496.

28. Fuster, V., D. N. Fass, M. P. Kaye, M. Jusa, A. R. Zinsmeister, and E. J. W. Bowie. 1982. Arterisclerosis in normal and von Willebrand pigs: longterm prospective study and aortic transplantation study. Circ. Res. 51:587-593.

29. Hironaka, T, Y. Ikari, Y. Miyata, S. Morimoto, and A. Tsunoo. 1984. Transplantation of extensor carpi radialis longus muscle in normal and dystrophic chicks. Exp. Neurol. 83:392-402.

30. Pabico, R. C., B. A. McKenna, and R. B. Freeman. 1975. Renal function before and after unilateral nephrectomy in renal donors. Kidney Int. 8:166-175. 
31. Nesbitt, T., B. Lobaugh, and M. K. Drezner. 1987. Calcitonin stimulation of renal 25 -hydroxyvitamin D-1 $\alpha$-hydroxylase activity in hypophosphatemic mice. Evidence that the regulation of calcitriol production is not universally abnormal in X-linked hypophosphatemia. J. Clin. Invest. 79:15-19.

32. Nesbitt, T., G. A. Davidai, and M. K. Drezner. 1988. Abnormal adenosine 3'5'-monophosphate stimulation of renal 1,25-dihydroxyvitamin D production in Hyp- mice: evidence that 25 -hydroxyvitamin D-1 $\alpha$-hydroxylase dysfunction results from aberrant intracellular function. Endocrinology. 124:1184-1189.

33. Nesbitt, T., and M. K. Drezner. 1990. Abnormal parathyroid hormonerelated peptide stimulation of renal 25-hydroxyvitamin D-1-hydroxylase in Hypmice: evidence for a generalized defect of enzyme activity in the proximal convoluted tubule. Endocrinology. 127:843-848.

34. Posillico, J. T., B. Lobaugh, L. H. Muhlbaier, and M. K. Drezner. 1985 Abnormal parathyroid function in the X-linked hypophosphatemic mouse. $\mathrm{Cal}$ cif. Tissue Int. 37:418-422.

35. Kiebzak, G. M., and R. A. Meyer, Jr. 1981. X-linked hypophosphatemic mice respond to parathyroidectomy. Miner. Electrolyte Metab. 6:153-164.

36. Drezner, M. K. 1990. Tumor-associated rickets and osteomalacia. In Primer on Metabolic Bone Diseases and Disorders of Mineral Metabolism. M. J. Favus, editor. Am. Soc. Bone and Min. Res. Kelseyville, CA. 184-188.

37. Aschinberg, L. C., L. M. Soloman, P. M. Zeis, P. Justice, and I. M. Ro- senthal. 1977. Vitamin D-resistant rickets associated with epidermal nevus syndrome: Demonstration of a phosphaturic substance in the dermal lesions. $J$. Pediatr. 91:56-60.

38. Miyauchi, A., M. Fukase, M. Tsutsumi, and T. Fujita. 1988. Hemangiopericytoma-induced osteomalacia: Tumor transplantation in nude mice causes hypophosphatemia and tumor extracts inhibit renal 25-hydroxyvitamin D-1-hydroxylase activity. J. Clin. Endocrinol. Metab. 67:46-53.

39. Morgan, J. M., W. L. Hawley, A. I. Chenoweth, W. J. Retan, and A. G. Diethelm. 1974. Renal transplantation and hypophosphatemia with vitamin Dresistant rickets. Arch. Intern. Med. 134:549-552.

40. Rosenbaum, R. W., K. A. Hruska, A. Korkor, C. Anderson, and E. Slatopolsky. 1981. Decreased phosphate reabsorption after renal transplantation: Evidence for a mechanism independent of calcium and parathyroid hormone. Kidney Int. 19:568-578.

41. Fukase, M., L. V. Avioli, S. J. Birge, and L. R. Chase. 1984. Abnormal regulation of 25 -hydroxyvitamin $\mathrm{D}_{3}-1 \alpha$-hydroxylase activity by calcium and calcitonin in renal cortex from hypophosphatemic (Hyp) mice. Endocrinology. 114:1203-1207.

42. Ecarot-Charrier, B., F. H. Glorieux, R. Travers, M. Desbarats, F. Bouchard, and A. Hink. 1988. Defective bone formation by transplanted Hyp-mouse bone cells into normal mice. Endocrinology. 123:768-773. 